\title{
Polymorphism of winter phenotype in Siberian hamster: consecutive litters do not differ in photoresponsiveness but prolonged acclimation to long photoperiod inhibits winter molt
}

\author{
Anna S. Przybylska-Piech ${ }^{1 *}$ (D), Michał S. Wojciechowski ${ }^{1}$ (D) and Małgorzata Jefimow ${ }^{2}$ (D)
}

\begin{abstract}
Background: The theory of delayed life history effects assumes that phenotype of adult individual results from environmental conditions experienced at birth and as juvenile. In seasonal environments, being born late in the reproductive season affects timing of puberty, body condition, longevity, and fitness. We hypothesized that lateborn individuals are more prone to respond to short photoperiod (SP) than early born ones. We used Siberian hamsters Phodopus sungorus, a model species characterized by high polymorphism of winter phenotype. We experimentally distinguished the effect of litter order (first or third) from the effect of exposure to long photoperiod (LP) before winter (3 months or 5 months) by manipulating the duration of LP acclimation in both litters. We predicted that, irrespective of the litter order, individuals exposed to long photoperiod for a short time have less time to gather energy resources and consequently are more prone to developing energy-conserving phenotypes. To assess effect of litter order, duration of acclimation to long days, and phenotype on basal cost of living we measured basal metabolic rate (BMR) of hamsters.
\end{abstract}

Results: Individuals born in third litters had faster growth rates and were bigger than individuals from first litters, but these differences vanished before transfer to SP. Litter order or duration of LP acclimation had no effects on torpor use or seasonal body mass changes, but prolonged acclimation to LP inhibited winter molting both in first and third litters. Moreover, individuals that did not molt had significantly higher BMR in SP than those which molted to white fur. Although one phenotype usually predominated within a litter, littermates were often heterogeneous. We also found that over $10 \%$ of individuals presented late response to short photoperiod.

Conclusions: Our data indicate that duration of postnatal exposure to LP may define propensity to photoresponsiveness, regardless of the litter in which animal was born. Existence of littermates presenting different phenotypes suggests a prudent reproductive strategy of investing into offspring of varied phenotypes, that might be favored depending on environmental conditions. This strategy could have evolved in response to living in stochastic environment.

Keywords: Delayed life history effect, Winter phenotype, Polymorphism, Torpor, Molting, Metabolism

\footnotetext{
* Correspondence: annprz@umk.pl

'Department of Vertebrate Zoology and Ecology, Nicolaus Copernicus University, Toruń, Poland

Full list of author information is available at the end of the article
}

\section{$\triangle B M C$}

(c) The Author(s). 2021 Open Access This article is licensed under a Creative Commons Attribution 4.0 International License, which permits use, sharing, adaptation, distribution and reproduction in any medium or format, as long as you give appropriate credit to the original author(s) and the source, provide a link to the Creative Commons licence, and indicate if changes were made. The images or other third party material in this article are included in the article's Creative Commons licence, unless indicated otherwise in a credit line to the material. If material is not included in the article's Creative Commons licence and your intended use is not permitted by statutory regulation or exceeds the permitted use, you will need to obtain permission directly from the copyright holder. To view a copy of this licence, visit http://creativecommons.org/licenses/by/4.0/ The Creative Commons Public Domain Dedication waiver (http://creativecommons.org/publicdomain/zero/1.0/) applies to the data made available in this article, unless otherwise stated in a credit line to the data. 


\section{Background}

The ability to respond to day length (photoperiodism) allows animals to change phenotype across the annual cycle. Response to shortening photoperiod consists of morphological, physiological and behavioral adjustments and results in development of winter phenotype. In winter, small mammals, which are mostly long-day breeders, regress gonads and cease reproduction [1-3], decrease body mass $\left(m_{\mathrm{b}}\right)$ [4-6], some molt to white fur [7-10], and heterothermic species use torpor $[11,12]$. These adjustments allow for energy savings and are considered beneficial for winter survival, yet individuals insensitive to changes in day length (nonresponding individuals), or individuals presenting only some of winter traits (partial-responding individuals) also exist in many populations $[7,8,13-20]$. The diversity of winter phenotypes may result from complexity of physiological and molecular mechanisms underlying photoresponsiveness [21, 22]. Melatonin, the hormonal signal of day length, enters multiple molecular pathways which control molting, torpor expression or gonadal regression [23, 24]. Although these pathways are often interrelated, winter traits may be regulated independently $[23$, 25-27].

According to the theory of delayed life history effects, phenotype of an adult individual results from environmental conditions experienced at birth, and later during growth and maturation [28-30]. Thus, it may be also affected by the time of birth during the reproductive season [31-33]. Late- and early born individuals differ in time it takes to reach puberty $[34,35]$, strategy of winter survival [33, 35], and longevity [36, 37]. Individuals born later during the reproductive season are often smaller $[32,38]$, grow slower [33] and have a lower probability of winter survival [36] than individuals born earlier. One can argue that late-born individuals have less time to grow and gather energy reserves before winter [32, 38].

In the Boreal and Temperate Zones seasonally changing day length correlates with changes in ambient temperature and resource availability. Day length experienced during development influences responsiveness to short day in adult Siberian hamsters Phodopus sungorus, a long-day breeder, which is a model animal in the studies of seasonal adjustments in physiology [16, 17, 39]. A majority of hamsters born and/or weaned under photoperiod shorter than $15 \mathrm{~h}$ respond to short days [17, 33], contrary to those born or weaned under $16 \mathrm{~h}$ photoperiod or longer $[14,15,33]$. Because photoperiod is related to time of the year, it has been proposed that nonresponsiveness to short days results from being born early in the reproductive season and exposure to long days during first weeks of life [17]. Butler et al. [33, 40] used simulated natural photoperiod to demonstrate that the proportion of nonresponding Siberian hamsters was greater in cohorts born under lengthening photoperiod (early born cohorts) than in cohorts born when days were shortening (late-born cohorts). In all cohorts responders always predominated, but even among hamsters born late in the season over $10 \%$ of individuals did not respond to short days [33]. This suggests that factors other than day length may play a role in development of the nonresponding phenotype.

Between April and September Siberian hamsters may deliver up to 5 or 6 litters [41], therefore photoperiod experienced by offspring at birth and during early development may considerably differ. Since being born late in the season is intrinsically related to shorter exposure to long photoperiod, it is hard to disentangle the effect of photoperiod and litter order on adult winter phenotype. In many mammalian species, both long- and short-day breeders, litters differ in offspring quality [42-45]. Depending on the species, subsequent litters can be bigger [44-46] or smaller [43, 47] or not different from each other $[48,49]$.

We proposed that extrinsic factors, such as access to energy resources prior to winter, influence the strategy of winter survival in small long-day breeding mammals and hypothesized that being born late in the reproductive season increases probability of subsequent development of photoresponsiveness. We aimed to experimentally distinguish the effect of litter order from the effect of exposure to long photoperiod (LP) before winter by manipulating the duration of LP acclimation in consecutive litters (Fig. 1). We predicted that the proportion of individuals developing traits characteristic for an energy-conserving phenotype (white fur, torpor use, and low $m_{\mathrm{b}}$ ) would be greater among individuals exposed to long photoperiod for a short time, irrespective of the litter in which they were born. As a model we used the Siberian hamster, photosensitive rodent that exhibits a high level of polymorphism of winter phenotype, from responding individuals, through individuals which develop only some of winter traits, to nonresponding ones [13, 50, 51]. Additionally, we compared basal metabolic rate (BMR) of animals from different experimental groups to assess effect of litter order, age, photoperiod and winter phenotype traits on basal energy consumption.

\section{Results \\ Effect of litter order and duration of LP acclimation on offspring body mass}

Offspring $m_{\mathrm{b}}$ increased with dam $m_{\mathrm{b}}$ (LME: $\mathrm{F}(1,23)=5.951$, $P=0.026)$ and was negatively correlated with litter size, but the latter was true only in first litters (litter order $\times$ litter size, LME: $\mathrm{F}(1,372)=5.978, P=0.015)$. Offspring had similar $m_{\mathrm{b}}$ for the first nine days of life, irrespective of litter order, but thereafter offspring from first litters were smaller than third litter individuals (litter order $\times$ age, LME: $\mathrm{F}(1,351)=5.861$, $P<0.001$; Fig. 2). Although differences in body mass 


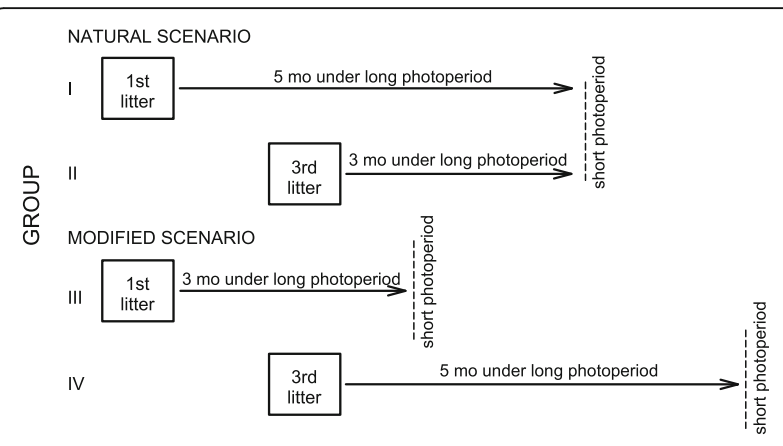

Fig. 1 Experimental design. Siberian hamsters were divided into four groups differing in time of birth (1st or 3rd litters) and duration of acclimation to long photoperiod (3 or 5 months). See

Methods for details

between consecutive litters ranged from $9.56 \%$ at day 12 of life to $16.43 \%$ at day 15 , it vanished between day 45 and day 90. However 60-day old hamsters from third litters were bigger than individuals from first litters (litter order $\times$ age, LME: $F(1,600)=4.785, P=0.003$, Fig. 2). Between days 45 and 90 males from first and third litters did not differ but females born in first litter were smaller than those born in third litter (litter order $\times$ sex, LME: $\mathrm{F}(1,485)=6.581, P=0.011$ ). Body mass of offspring acclimated to LP for 3 or 5 months did not differ prior to transfer to SP (GLM: $F(1,199)=1.608, P=$ 0.206). Model designs and results of the analysis of variance are given in Table 1, whereas data on hamster $m_{\mathrm{b}}$ are given in Table 2.

\section{Parental effect on offspring phenotype}

None of the parental pairs delivered offspring which developed only one phenotype (the same set of winter traits) in response to short photoperiod. In some pairs,

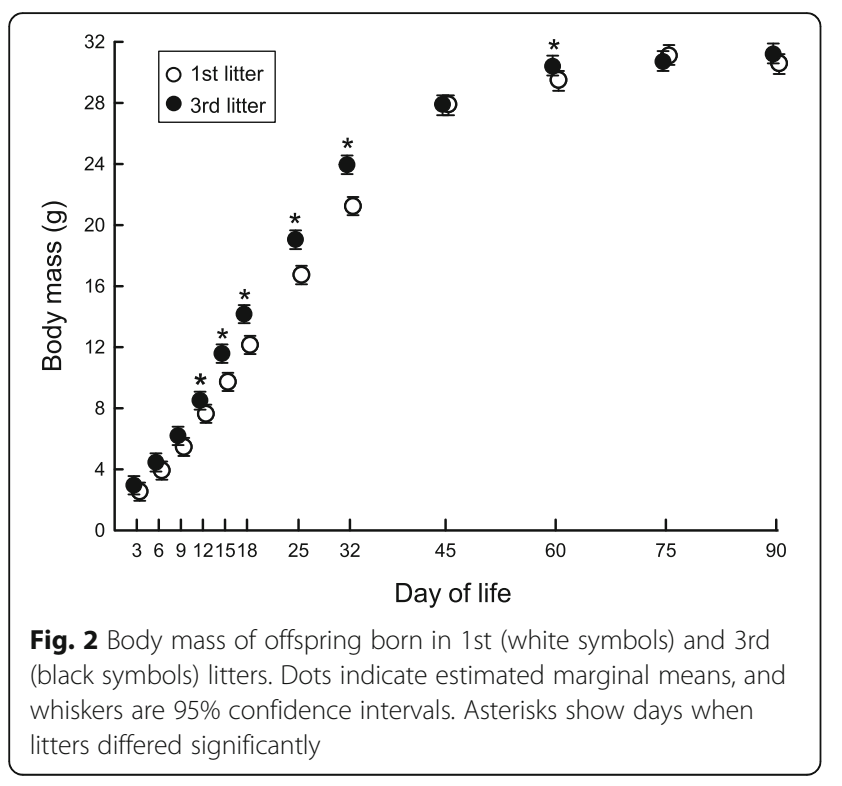

most offspring turned white and used torpor, whereas in other pairs grey offspring that did not use torpor predominated. Different phenotypes among littermates were more common in first (in 24 out of 25 parental pairs) than in third litters (in 17 out of 25 parental pairs) $\left(x^{2}(1\right.$, $50)=6.640, P=0.010$ ). For example, in three parental pairs littermates showed all possible combinations of winter traits, and therefore phenotypes: white fur and torpor use, white fur without torpor use, grey fur and torpor use, and grey fur without torpor use. In another 14 pairs, offspring presented three different phenotypes and in 8 pairs only two different combinations of traits. Because we did not know parental phenotype, we were not able to calculate heritability of winter traits. However, the goodness of fit of models with and without random effect of parental ID differed significantly $(P=$ $0.001)$, suggesting a strong parental effect on offspring phenotype.

\section{Effect of litter order and duration of LP acclimation on photoresponsiveness}

During the first 16 weeks of acclimation to SP, 94 out of 200 animals used daily torpor and 125 animals molted to white fur. Between weeks 20 and 37 of acclimation to SP, another 23 animals molted and 29 entered torpor for the first time. Generally, experimental groups did not differ in propensity to use torpor (litter order $\times$ duration of LP acclimation $\chi^{2}(1$, $200)<0.001, P=0.993$ ) or molting (litter order $\times$ duration of LP acclimation $X^{2}(1,200)<0.001, P=$ $0.988)$. Neither litter order $\left(\chi^{2}(2,200)=0.194, P=\right.$ $0.907)$ nor duration of LP acclimation $\left(\chi^{2}(2,200)=\right.$ 1.660, $P=0.436$; Table 3) affected the use of torpor. There was also no effect of litter order on molting $\left(\mathrm{X}^{2}(2,200)=0.128, P=0.938 ;\right.$ Table 3$)$. However, in groups acclimated to LP for 3 months we observed $71 \%$ individuals which molted to white in response to SP, and only $54 \%$ in groups acclimated to LP for 5 months $\left(x^{2}(2,200)=6.36, P=0.041\right.$; Table 3$)$. In the latter groups, we observed also almost two times more late-responding individuals than in groups acclimated to LP for 3 months (Table 3).

The higher the initial $m_{\mathrm{b}}$ of hamsters at the end of acclimation to long days, the greater the decrease of $m_{\mathrm{b}}$ after acclimation to short days (GLM: $F(1,198)=14.82$, $P<0.001)$. Neither litter order (GLM: $F(1,186)=1.287$, $P=0.258)$ nor duration of LP acclimation (GLM: F $(1$, $186)=1.345, P=0.248)$ affected $m_{\mathrm{b}}$ changes after 16 weeks in SP. We found that $m_{\mathrm{b}}$ change correlated with other winter phenotype traits. Namely, individuals using torpor (regardless of the time spent in SP) lost between 1 and $9 \%$ of initial $m_{\mathrm{b}}$ while individuals that did not use torpor maintained initial $m_{\mathrm{b}}$ or even gained it to $3 \%$ (GLM: $\mathrm{F}(1,186)=11.134, P<0.001)$. Changes of $m_{\mathrm{b}}$ in 
Table 1 Results of the type III analysis of variance calculated for offspring body mass in Siberian hamster

\begin{tabular}{|c|c|c|c|c|c|}
\hline Model no & Trait & Model & Factors & $F(d f)$ & P-value \\
\hline \multirow[t]{6}{*}{1} & \multirow[t]{6}{*}{$m_{b}$ until 32-day of life } & \multirow[t]{6}{*}{ LME } & age & $633.54(7,55)$ & 0.006 \\
\hline & & & litter order & $3.49(1,374)$ & 0.062 \\
\hline & & & litter size & $9.45(2,283)$ & 0.002 \\
\hline & & & $\operatorname{dam} m_{\mathrm{b}}$ & $5.95(1,23)$ & 0.026 \\
\hline & & & age $\times$ litter order & $5.86(7,351)$ & $<0.001$ \\
\hline & & & litter order $\times$ litter size & $5.98(1,372)$ & 0.015 \\
\hline \multirow[t]{5}{*}{2} & \multirow{5}{*}{$\begin{array}{l}m_{\mathrm{b}} \text { between } 45 \text { and } \\
90 \text {-day of life }\end{array}$} & \multirow[t]{5}{*}{ LME } & age & $108.05(3,601)$ & $<0.001$ \\
\hline & & & litter order & $0.58(1,188)$ & 0.447 \\
\hline & & & sex & $318.83(1,485)$ & $<0.001$ \\
\hline & & & age $\times$ litter order & $4.78(3,601)$ & 0.003 \\
\hline & & & litter order $\times$ sex & $3.58(1,485)$ & 0.011 \\
\hline \multirow[t]{3}{*}{3} & \multirow[t]{3}{*}{$m_{\mathrm{b}}$ prior to transfer to SP } & \multirow[t]{3}{*}{ GLM } & litter order & $2.44(1,199)$ & 0.120 \\
\hline & & & duration of LP acclimation & $1.61(1,199)$ & 0.206 \\
\hline & & & sex & $136.26(1,199)$ & $<0.001$ \\
\hline \multirow[t]{8}{*}{4} & \multirow[t]{8}{*}{$m_{b}$ after 16 weeks under SP } & \multirow[t]{8}{*}{ GLM } & litter order & $0.81(1,186)$ & 0.368 \\
\hline & & & duration of LP acclimation & $1.55(1,186)$ & 0.214 \\
\hline & & & sex & $0.34(1,186)$ & 0.563 \\
\hline & & & torpor use & $17.02(2,186)$ & $<0.001$ \\
\hline & & & molting & $81.71(2,186)$ & $<0.001$ \\
\hline & & & initial mb & $11.10(1,186)$ & 0.001 \\
\hline & & & sex $\times$ torpor use & $4.58(2,186)$ & 0.034 \\
\hline & & & sex $\times$ molt & $9.45(2,186)$ & 0.002 \\
\hline
\end{tabular}

$m_{b}$ body mass, SP short photoperiod, LP long photoperiod, LME linear mixed effect model, GLM general linear model. Significant effects are indicated in bold

different molting categories were related to sex $(\operatorname{sex} \times$ molting GLM: $\mathrm{F}(1,186)=5.721, P=0.004)$. Both, grey males and grey females gained up to $4.5 \%$ of initial $m_{\mathrm{b}}$, whereas white males and white females lost between 9 and $14 \%$ of initial $m_{\mathrm{b}}$. Within animals that molted after 20 weeks under SP, males gained around $5 \%$, and females lost over $5 \%$ of initial $m_{\mathrm{b}}$.

Changes of $m_{\mathrm{b}}$ during further acclimation to SP in late responding animals correlated with their phenotype. Animals that molted within 16 weeks in SP and started to use torpor later than 20 weeks in SP did not change their $m_{\mathrm{b}}$ any further. Most individuals that both molted and started to use torpor later than after 20 weeks in SP decreased their $m_{\mathrm{b}}$ by approximately $16.3 \pm 10.2 \%$ (Fig. 3) but some of them also gained $m_{\mathrm{b}}$ by $10.1 \pm 7.6 \%$. The two grey individuals that started to use torpor later than after 20 weeks in SP differed between each other, one lost $m_{\mathrm{b}}$ while the other maintained constant $m_{\mathrm{b}}$.

\section{Basal metabolic rate}

Basal metabolic rate was repeatable in 3-month-old animals $(\tau=0.263, P=0.001)$ and between acclimation to long photoperiod and short photoperiod $(\tau=0.235, P=$
0.001), but not in late responders between subsequent measurements $(\tau=0.060, P=0.151)$.

Hamsters born in first or third litters did not differ in BMR at the age of 3 months (LMM: $F(1,217)=1.486$, $P=0.224)$ but after adjusting for $m_{\mathrm{b}}$ females had higher BMR than males $(0.273 \pm 0.002 \mathrm{~W}$ and $0.261 \pm 0.002 \mathrm{~W}$ respectively; LMM: $\mathrm{F}(1.224)=15.218, P<0.001)$. Basal metabolic rate decreased from $0.261 \pm 0.004 \mathrm{~W}$ in long photoperiod to $0.247 \pm 0.004 \mathrm{~W}$ after 16 weeks of acclimation to short photoperiod (LMM: $F(1.591)=66.739$, $P<0.001$, Fig. 4). This decrease depended on molting category, litter order and duration of LP acclimation. Namely, individuals acclimated to long days for 3 months did not change BMR between photoperiods, whereas those acclimated to LP for 5 months decreased BMR after being transferred to short days (photoperiod $\times$ duration of LP acclimation LMM: $F(1,595)=44.234$, $P<0.001)$. While all individuals had similar BMR in long photoperiod, after 16 weeks under short photoperiod BMR did not differ only among hamsters from third litters. Among hamsters from first litters, grey individuals had $6-10 \%$ higher BMR than white ones, and also than individuals that molted to white fur later $($ photoperiod $\times$ litter order $\times$ molting; LMM: $F(1,590)=$ 
Table 2 Estimated marginal means \pm SE for body mass of offspring from first and third litters, calculated from models one and two (Table 1.), compared pairwise with Tukey's HSD test adjusted for multiple comparisons. Significant differences are indicated in bold

\begin{tabular}{|c|c|c|c|}
\hline \multirow{2}{*}{$\begin{array}{l}\text { Day } \\
\text { of } \\
\text { life } \\
\end{array}$} & \multicolumn{2}{|c|}{ Body mass (g) } & \multirow{2}{*}{$\begin{array}{l}\text { Post-hoc } \\
P \text {-value }\end{array}$} \\
\hline & $1^{\text {st }}$ litter & $3^{\text {rd }}$ litter & \\
\hline 3 & $2.54 \pm 0.30$ & $2.95 \pm 0.30$ & 0.274 \\
\hline 6 & $3.91 \pm 0.30$ & $4.45 \pm 0.30$ & 0.159 \\
\hline 9 & $5.46 \pm 0.30$ & $6.19 \pm 0.30$ & 0.055 \\
\hline 12 & $7.63 \pm 0.30$ & $8.50 \pm 0.29$ & 0.021 \\
\hline 15 & $9.72 \pm 0.30$ & $11.57 \pm 0.30$ & 0.001 \\
\hline 18 & $12.14 \pm 0.30$ & $14.16 \pm 0.30$ & 0.001 \\
\hline 25 & $16.74 \pm 0.31$ & $19.05 \pm 0.31$ & 0.001 \\
\hline 32 & $21.24 \pm 0.30$ & $23.95 \pm 0.31$ & 0.001 \\
\hline 45 & $27.90 \pm 0.33$ & $27.90 \pm 0.33$ & 0.998 \\
\hline 60 & $29.50 \pm 0.33$ & $30.40 \pm 0.33$ & 0.042 \\
\hline 75 & $31.10 \pm 0.33$ & $30.70 \pm 0.33$ & 0.437 \\
\hline 90 & $30.60 \pm 0.33$ & $31.20 \pm 0.33$ & 0.174 \\
\hline
\end{tabular}

Significant differences are indicated in bold

5.376, $P=0.005$, Fig. 4). In late responding first litter individuals BMR increased in the course of acclimation to short photoperiod, whereas in individuals from third litters BMR was the same between measurements in short photoperiod (litter order $\times$ time of measurement LMM: $\mathrm{F}(1,139)=3.615, P=0.029)$. Model designs and results of the analysis of variance are given in Table 4.

\section{Discussion}

Early life events may profoundly affect adult phenotype [31-33]. We found that individuals that spent 3 months under long photoperiod were more prone to molt to a white fur than individuals from groups acclimated to long photoperiod for 5 months (Table 3). Additionally, white individuals had lower basal energy expenditure in short photoperiod than grey ones (Fig. 4). This supports our prediction that individuals exposed to long photoperiod for a short time, irrespective of the litter in which they were born, were more prone to develop an energyconserving phenotype. However, our prediction that individuals born later during reproductive season have less time to gather energy reserves before winter was not supported because initial differences in body mass between hamsters originating from consecutive litters vanished before transfer to short photoperiod (Fig. 2). We also found that litter order or duration of LP acclimation had no effect on torpor use or $m_{\mathrm{b}}$ change.

\section{Delayed life history effect and offspring body mass}

In the present study, consecutive litters differed in growth rate and individuals born in third litters grew faster than individuals born in first litters (Fig. 2, Table 2). In many mammalian species, including Siberian hamsters, litter order or time of birth during reproductive season affect litter or offspring quality [42-44, 46, 47]. It is true both for long- and short-day breeders. Long-day breeders, which are mostly small mammals with short gestation periods, mate in spring, while short-day breeders, like large ungulates, mate in autumn and have long gestation period. Although these groups differ in gestation lengths, parturition takes place at the time of year with highest resource availability. Therefore, late birth during the reproductive season may affect quality of mammalian offspring. Offspring of short-day breeder, red deer Cervus elaphus, born late during reproductive season were smaller than early born ones [36]. In European rabbits Oryctolagus cuniculus offspring from subsequent litters [47] were smaller than offspring born in earlier litters, whereas in garden dormice Eliomys quercinus [52], Uinta ground squirrels Spermophilus armatus [46], or Siberian hamsters [44] this trend was opposite. Differences in $m_{\mathrm{b}}$ between consecutive litters might have resulted from the efficiency of parental care, dam's age, and body condition. In European rabbits previous reproductive activity might have had negative effect on

Table 3 Effect of litter order and duration of long photoperiod (LP) acclimation on torpor use and molting in Siberian hamsters born in first or third litters, and acclimated to LP for 3 or 5 months. Each group consisted of 50 individuals acclimated to the short day for 16 weeks. Bold text indicates statistically significant differences $(P<0.05)$ between groups acclimated to LP for 3 or 5 months

\begin{tabular}{|c|c|c|c|c|c|c|c|}
\hline \multirow[b]{3}{*}{$\begin{array}{l}\text { Duration of LP acclimation } \\
\text { (months) }\end{array}$} & \multirow[b]{3}{*}{ Litter } & \multicolumn{3}{|c|}{ Torpor use } & \multicolumn{3}{|l|}{ Molting } \\
\hline & & \multicolumn{3}{|c|}{ Number of individuals } & \multicolumn{3}{|c|}{ Number of individuals } \\
\hline & & $\begin{array}{l}\text { using } \\
\text { torpor }\end{array}$ & $\begin{array}{l}\text { not using } \\
\text { torpor }\end{array}$ & $\begin{array}{l}\text { using torpor after } 20 \text { weeks } \\
\text { under SP }\end{array}$ & $\begin{array}{l}\text { molting to } \\
\text { white }\end{array}$ & $\begin{array}{l}\text { remaining } \\
\text { grey }\end{array}$ & $\begin{array}{l}\text { molting to white after } 20 \\
\text { weeks in SP }\end{array}$ \\
\hline \multirow[t]{2}{*}{3} & 1 & 24 & 20 & 6 & 34 & 12 & 4 \\
\hline & 3 & 27 & 17 & 6 & 37 & 9 & 4 \\
\hline \multirow[t]{2}{*}{5} & 1 & 22 & 20 & 8 & 28 & 15 & 7 \\
\hline & 3 & 21 & 20 & 9 & 26 & 16 & 8 \\
\hline
\end{tabular}


white individual using torpor

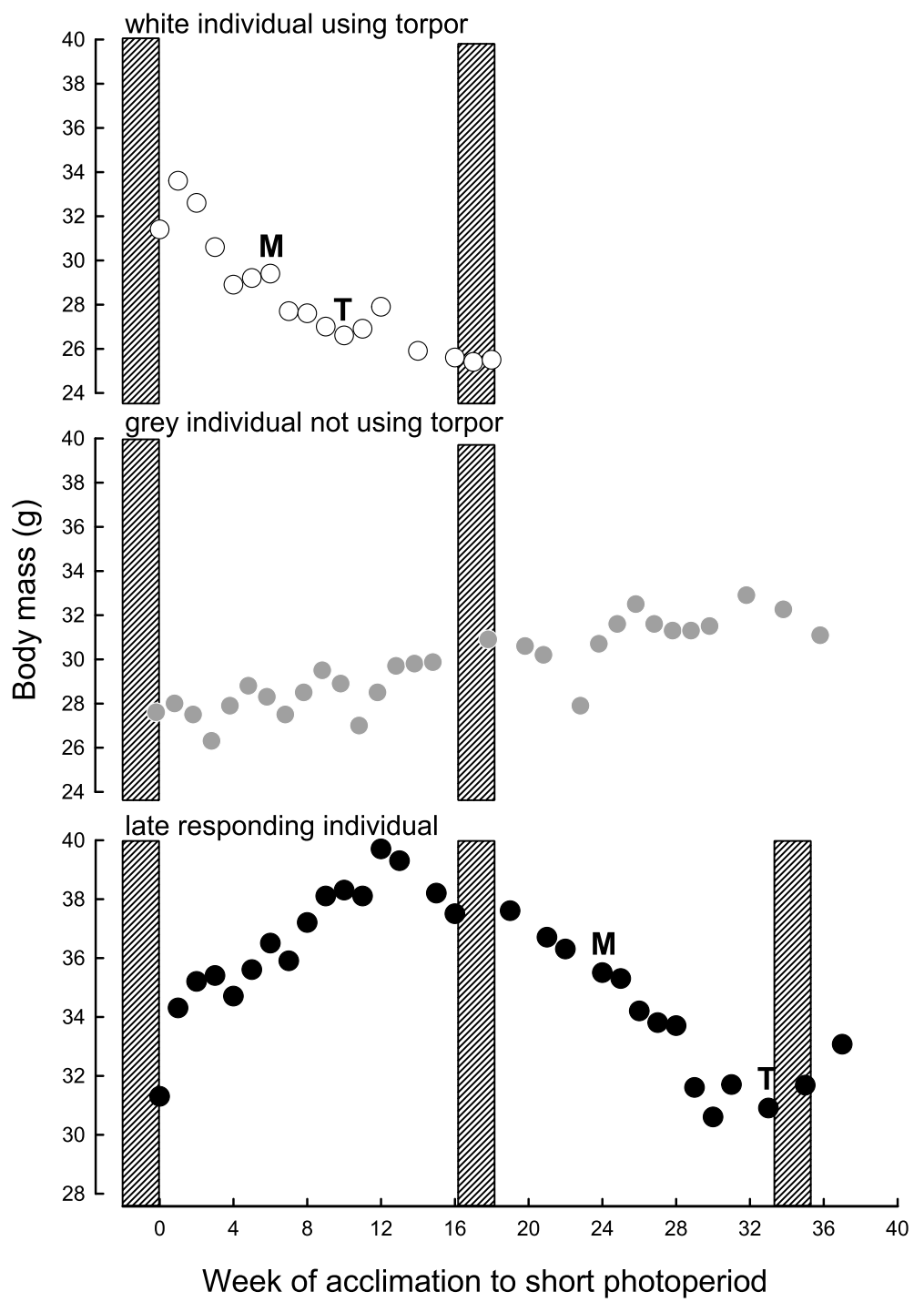

Fig. 3 Changes in body mass in representative white individual using torpor (top panel), grey individual not using torpor (middle panel) and individual using torpor and molt to white fur later than after 20 weeks under short photoperiod (SP) (bottom panel). White individuals using torpor were transferred to LP after BMR measurements in SP, and there are no data points afterward. Hatched bars indicate time of BMR measurements. $M$ - stage 3 of molting; $T$ - first episode of torpor

current reproduction since first litters had higher growth rate than consecutive ones [47]. Conversely, laboratory mice [53] and rats [54] increased lactation performance and therefore reproductive effectiveness with successive litters. However, increase in reproductive effectiveness was limited to first two or three litters, and then it constantly decreased $[55,56]$. Quality of offspring might be related also to maternal age and body condition $[55,57]$. Bigger dams of both long- and short-day breeders delivered bigger offspring [58-60], but reproductive effectiveness depended also on time of the year when the dam was born [55].

In a previous study we found that offspring $m_{\mathrm{b}}$ in $\mathrm{Si}$ berian hamsters was related to parental phenotype [44] and nonresponding hamsters delivered bigger offspring than responding ones. Since responding individuals regress gonads, energy allocated into gonadal recrudescence and rebuilding of body reserves may reduce resources available for reproduction. Presumably this is why photoresponding hamsters commenced breeding later than nonresponding ones [44]. Regardless of parental phenotype offspring from successive litters were always bigger than earlier ones ([44], present study). In the present study, a negative relationship between litter size and offspring $m_{\mathrm{b}}$ only in first litters may suggest that after winter all individuals, regardless of their photoresponsiveness and gonadal status, might be in negative energy balance. It clearly indicates that 


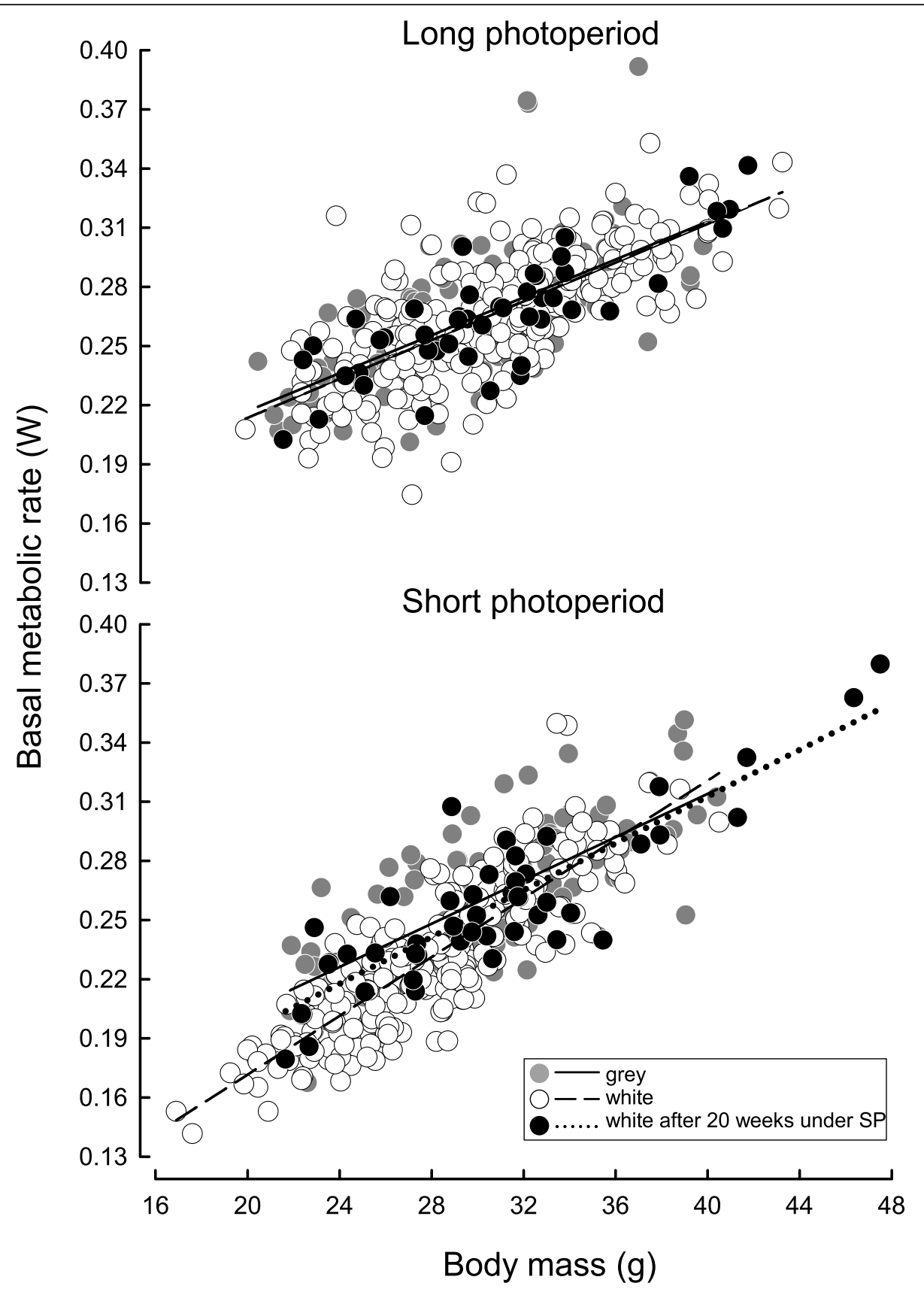

Fig. 4 Relationship between basal metabolic rate (BMR) and body mass in white and grey individuals and in individuals that molted to white after 20 weeks under short photoperiod. Top panel shows data recorded in long photoperiod prior transfer to short photoperiod, and bottom panel shows data recorded after 16 weeks in short photoperiod. Regression lines did not differ between groups in long photoperiod but did differ in short photoperiod (see text). BMR was measured at $\sim 28.5^{\circ} \mathrm{C}$

reproduction-related trade-offs appear only when energy resources are limited.

We predicted that individuals born later in the reproductive season would develop traits of an energyconserving phenotype, because these individuals have less time for gathering energy reserves, and they are considered to have lower chance to survive and reproduce next year $[34,38,61]$. On the one hand faster growth rate of offspring from subsequent litters could have compensated for late birth $[46,62,63]$. On the other hand, compensatory growth might have delayed consequences, such as inhibited growth in adults [64-66]. Since differences in $m_{\mathrm{b}}$ between litters vanished already at day 45 of life (Fig. 2), the latter explanation may be the case in Siberian hamsters. Under natural conditions, this species breeds from April to September [41]. Even if dams give birth every 23- 
Table 4 Results of the type III analysis of variance calculated for basal metabolic rate (BMR) in Siberian hamster

\begin{tabular}{|c|c|c|c|c|c|}
\hline Model no & Trait & Model & Factors & $F(d f)$ & P-value \\
\hline \multirow[t]{4}{*}{1} & \multirow[t]{4}{*}{ BMR in 3 months old animals } & \multirow[t]{4}{*}{ LME } & $m_{\mathrm{b}}$ & $189.79(1,222)$ & $<0.001$ \\
\hline & & & litter order & $1.49(1,208)$ & 0.224 \\
\hline & & & sex & $15.22(1,224)$ & $<0.001$ \\
\hline & & & $m_{b} \times$ litter order & $2.87(1,209)$ & 0.091 \\
\hline \multirow[t]{13}{*}{2} & \multirow[t]{13}{*}{ BMR after 16 weeksunder SP } & \multirow[t]{13}{*}{ LME } & $m_{\mathrm{b}}$ & $715.89(1,412)$ & $<0.001$ \\
\hline & & & litter order & $0.10(1,191)$ & 0.752 \\
\hline & & & duration of LP acclimation & $0.37(1,190)$ & 0.542 \\
\hline & & & sex & $24.43(1,274)$ & $<0.001$ \\
\hline & & & photoperiod & $66.74(1,591)$ & $<0.001$ \\
\hline & & & molting & $9.07(2,190)$ & 0.001 \\
\hline & & & duration of LP acclimation $\times$ molting & $3.31(2,189)$ & 0.038 \\
\hline & & & duration of LP acclimation $\times$ photoperiod & $0.18(1,595)$ & 0.673 \\
\hline & & & litter order $\times$ molting & $4.61(2,190)$ & 0.011 \\
\hline & & & litter order $\times$ photoperiod & $2.92(1,590)$ & 0.087 \\
\hline & & & photoperiod $\times$ molting & $1.38(2,594)$ & 0.252 \\
\hline & & & duration of LP acclimation $\times$ photoperiod $\times$ molting & $0.35(2,591)$ & 0.707 \\
\hline & & & litter order $\times$ photoperiod $\times$ molting & $5.38(2,590)$ & 0.005 \\
\hline \multirow[t]{7}{*}{3} & \multirow[t]{7}{*}{ BMR of late responding individuals } & \multirow[t]{7}{*}{ LME } & $m_{\mathrm{b}}$ & $204.38(1,40)$ & $<0.001$ \\
\hline & & & litter order & $0.02(1,99)$ & 0.877 \\
\hline & & & duration of LP acclimation & $2.78(1,22)$ & 0.109 \\
\hline & & & sex & $7.67(1,24)$ & 0.011 \\
\hline & & & time of measurement & $6.23(2,137)$ & 0.002 \\
\hline & & & molting & $0.01(1,22)$ & 0.940 \\
\hline & & & litter order $\times$ time of measurement & $3.62(2,139)$ & 0.029 \\
\hline
\end{tabular}

$m_{b}$ body mass, SP short photoperiod, $L P$ long photoperiod, $L M E$ linear mixed effect model. Significant effects are indicated in bold

30 days [67], third litters are born in June or July, i.e. 2-3 months before winter. Since we did not find differences between litters in body mass or BMR at the age of 3 months and prior to transfer to SP, it suggests that this time is long enough to complete growth before winter.

\section{Delayed life history effect and photoresponsiveness}

We observed the whole spectrum of possible winter phenotypes among the study animals, including late responders that developed winter traits after more than 20 weeks under SP. It was previously reported that photoresponsiveness diminished with animal age and vanished after first year of life $[6,8]$. However, since hamsters from first and third litters were born $\sim 60$ days apart, age cannot explain differences in photoresponsiveness.

Being born late in reproductive season decreased probability of winter survival in hibernating rodents $[46,68]$. To our knowledge, direct effects of the duration of exposure to long photoperiod on physiology of seasonal rodents originating from early and late litters have not been tested before. Butler et al. [33, 40] found that more nonresponding hamsters were born in lengthening photoperiod (early cohorts), but nonresponding individuals were still present among individuals born under shortening photoperiod (late cohorts) [33, 40]. Nevertheless, the study did not report whether hamsters originated from first or consecutive litters.

Prolonged acclimation to LP reduced the number of individuals that molted to white fur in response to SP (Table 3). Seasonal molting is related to decreased prolactin level [21, 22]. Since Siberian hamsters born early during reproductive season may reach puberty before their first winter, prolonged acclimation to long photoperiod may increase prolactin level and therefore inhibit molting. Lack of molting can be regarded as disadvantageous because white fur increases insulation [69], but Boratyński et al. [51] did not find differences in thermal conductance between winter and summer acclimated Siberian hamsters. Although energy savings due to molting in small mammals are considered to be limited $[9,70]$, they may still be present. Here we found that after acclimation to short photoperiod, BMR of white animals was $10 \%$ lower than BMR of grey individuals (Fig. 4). Since high prolactin level has been shown 
to increase food intake, adipogenesis, and $m_{\mathrm{b}}[71,72]$ it may also explain high BMR of grey individuals acclimated to short days.

Neither litter order nor duration of LP acclimation affected torpor use or $m_{\mathrm{b}}$ change. As far as we know, this is first study which experimentally tested the effect of these factors on torpor use in Siberian hamsters. Torpor is an effective way to reduce energy expenditure under SP [73-75] and propensity for torpor increased in cold or after fasting $[76,77]$. It is plausible that we did not observe any effect of litter order and duration of LP acclimation on torpor use because of constant and relatively high ambient temperature during acclimation and food available ad libitum. This might also be a reason for similar $m_{\mathrm{b}}$ prior to transferring animals to SP and for similar time courses of $m_{\mathrm{b}}$ changes under SP in all groups. Although, individual body condition and energy reserves did not affect photoresponsiveness under mild conditions, it may be of great importance in harsh environments.

In the present study, prolonged acclimation to long days increased the number of late responding hamsters (Table 3). In these individuals the sequence of seasonal changes was the same as in typically responding ones (response after 12-16 weeks under SP). Namely, decrease in $m_{\mathrm{b}}$ was followed by molting and finally, occurrence of torpor (Fig. 3). However, late responders were in their winter phenotype for shorter time because photorefractoriness occurred at a similar time as during the typical response to SP $[11,78]$. Although the phenomenon of late responders is known $[11,78]$, the mechanism of delayed response to winter remains unexplained. It does not seem to be a laboratory artefact, because both abrupt and gradual changes from long to short days led to development of winter phenotypes and any differences vanished before the 12th week of SP acclimation [16, 79].

At first glance, existence of late responders under natural conditions seems to be difficult to understand. However, this picture may change when we account for the fact that other winter-related phenomena, such as snowfall or low ambient temperature, may be shifted towards spring. Since late responders from third litters were able to maintain low BMR for the entire course of acclimation to short photoperiod, their energy reserves might have been maintained for a longer time. Therefore, prolonged cold or unexpected snowfall in spring may favor a late winter response and explain maintenance of late responders in a population.

\section{Polymorphism in winter traits within a litter}

In the present study none of the parental pairs delivered offspring of one phenotype. Within a litter there were individuals of different responsiveness to SP, but in a few parental pairs one offspring phenotype predominated, suggesting heritability of the winter phenotype. Previous reports showed that phenotype was heritable in Siberian hamsters and white-footed mice $[3,14,80]$ and artificial selection led to increase of photoresponsiveness [81]. Since our colony is outbred, parental pairs were paired randomly and we did not control for their winter phenotype, calculation of heritability of winter traits was impossible. Polymorphism was more common in the first litters suggesting that individuals born earlier during the reproductive season may show higher variability in the response to shortening days, than less flexible, bigger and faster growing individuals from third litters. In our breeding colony some littermates that were maintained together in the same cage and under the same conditions developed different winter phenotypes (Fig. 5). According to Balanced Polyphenism Hypothesis [82, 83] offspring of different phenotypes may increase parental fitness, because each phenotype can be advantageous under specific environment conditions.

\section{Conclusions}

Winter phenotype may be considered as a suite of morphological, physiological and behavioral adjustments, but it seems that particular winter traits develop independently. To the best of our knowledge this is the first time when existence of different phenotypes among littermates is reported. Goldman and Goldman [15] proposed that all hamsters are born as responsive to short photoperiod but in some individuals this response is lost due

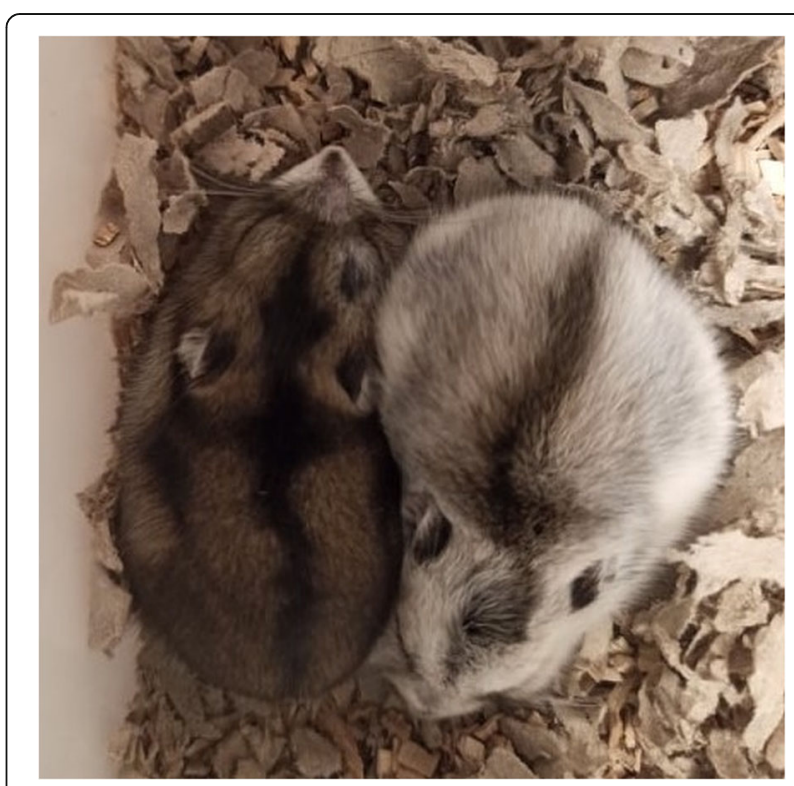

Fig. 5 Littermates of Siberian hamsters presenting different winter phenotypes. Left one remained grey and never used daily torpor, whereas right one molted to white and used daily torpor. Picture was taken after 14 weeks under short photoperiod (8L:16D) 
to extrinsic factors (e.g. long photoperiod during postnatal development). Indeed, time of birth during the season may affect strategy of winter survival since prolonged acclimation to long days inhibited seasonal molting and increased energy expenditure. Individuals which do not respond to SP and have higher BMR may have a lower chance to survive harsh winter than individuals which responded to SP with all physiological and morphological traits. Conversely, nonresponding individuals may develop a late response which may be favored under particular environmental condition. Although we did not find any delayed life history effects on torpor use or seasonal $m_{\mathrm{b}}$ changes, we suggest that under harsh environmental conditions and when resources are limited, or in younger individuals, energy supplies gathered before winter have significant effect on development of these winter traits and overall photoresponsiveness.

\section{Methods}

\section{Animals}

All experimental procedures were approved by the Local Committee for Ethics in Animal Research in Bydgoszcz, Poland (decisions: 8/2018, 22/2018, 48/2018, 18/2019). We used 200 Siberian hamsters, 100 males and $100 \mathrm{fe}-$ males, originating from our outbred colony maintained at the Faculty of Biological and Veterinary Sciences at the Nicolaus Copernicus University in Toruń, Poland. Half of animals were born in the first litters and another half in the third litters. All individuals were born between late May and late August. Litters were weaned at day 18 and each litter shared one cage until day 32. After that, we randomly selected 2 males and 2 females from each litter. Siblings of each sex were maintained together until they were two-months old. Thereafter each hamster was assigned to experimental group and transferred to an individual cage in which it was maintained throughout entire experiment. Groups did not differ in body mass at the age of 2 months (UNIANOVA $\mathrm{F}(1$, $200)=1.126, P=0.340$ ). To estimate growth rate, whole litters were weighed every 3 days until weaning and then every week until day 32 . Weighing was continued every two weeks until transfer to short photoperiod. After birth hamsters were maintained under long photoperiod (LP; $16 \mathrm{~L}: 8 \mathrm{D}$, lights on at 04:30 a.m.) at ambient temperature of $20 \pm 2{ }^{\circ} \mathrm{C}$. The $16 \mathrm{~h}$ day does not inhibit further response to short photoperiod [14, 17]. All animals were maintained in standard laboratory cages (Tecniplast, 1245 , Italy, $33 \times 20 \times 18 \mathrm{~cm}$ ) with deciduous wood chips as bedding material. Hamsters were fed with standard rodent food with higher content of protein and fat (60\% of carbohydrates, $10 \%$ of fat and $30 \%$ of protein; Labofeed H standard, Morawski, Kcynia, Poland) until the second month of life, and afterwards with standard maintenance food (67\% of carbohydrates, $8 \%$ of fat and
25\% of protein; Labofeed B standard, Morawski, Kcynia, Poland). Animals were supplied with drinking water ad libitum.

\section{Experimental protocol}

To determine the effect of time of birth on offspring photoresponsiveness, we set four experimental groups of 50 individuals each, which differed in the time of birth or in the duration of acclimation to long photoperiod after birth (Fig. 1). Although Siberian hamsters may breed immediately after parturition of previous litter [41, 84], a post-implantation embryonic diapause is common [67] and females usually deliver consecutive litters every $\sim 23-30$ days [44]. In our study third litters were $\sim 2$ months (50-65 days) younger than the first litters. Hamsters originating from first and third litters were randomly assigned to experimental groups which differed in duration of post-natal acclimation to long photoperiod. Two groups were intended to imitate natural conditions (Natural Scenario in Fig. 1). Group I was composed of animals born in first litters that were acclimated to LP for $\sim 5$ months and then transferred to short photoperiod (SP; $8 \mathrm{~L}: 16 \mathrm{D}$, lights on at 08:30 a.m.). Hamsters from Group II were born in third litters and since they were transferred to SP together with the first group, they were acclimated to LP for $\sim 3$ months. Groups III and IV were used to control for the effects of litter order and duration of LP acclimation on the development of winter traits (Modified Scenario in Fig. 1). Individuals from group III were born in the first litters, but their acclimation to LP was shortened to 3 months. In group IV, LP acclimation of animals born in the third litters was lengthened to $\sim 5$ months. To test our hypothesis, we used abrupt change of photoperiod, which allowed to precisely measure time spent in both photoperiodic regimes. Despite limitations discussed by Gorman et al. [16], this protocol has been widely used to induce a seasonal response in Siberian hamsters [7, 14, 85-89]. It has also been reported that proportion of nonresponding individuals was not related to the method of photoperiod transition from summer to winter [16]. To account for parental effect, all offspring were derived from 25 parental pairs. Each parental pair was represented by one male and one female offspring in each group, resulting in 8 offspring per parental pair across all groups.

\section{Determination of winter traits}

After transfer to SP, hamsters were maintained individually, in the same type of cages and at the same ambient temperature as in LP. Development of winter traits was determined after 16 weeks under SP based on pelage color, torpor use and $m_{\mathrm{b}}$ change. Traditionally, individuals were classified as responders, nonresponders, or partial responders based on set of several winter traits, 
such as daily torpor and pelage color or gonadal regression and $m_{\mathrm{b}}$ loss $[8,13,40,90]$. However, because winter traits are controlled by different hormonal and molecular pathways $[21,22]$ and they may develop independently in response to SP, such simple classifications become debatable. Here, we analyzed effect of delayed history effects on each winter trait separately. An individual was classified as using torpor if it entered at least one torpor episode (subcutaneous temperature $\leq 32{ }^{\circ} \mathrm{C}$, stereotypical posture, reduced responsiveness). To determine this, hamsters were injected subcutaneously in interscapular region with thermosensitive passive integrated transponders (BioTherm 13, Biomark, Boise, ID, USA) after 2 to 4 weeks of SP acclimation. Although the manufacturer-reported temperature range of transponders is $33^{\circ} \mathrm{C}$ to $43^{\circ} \mathrm{C}$, we calibrated them in a water bath against a high-precision mercury-in-glass thermometer between $27.0^{\circ} \mathrm{C}$ and $40.0^{\circ} \mathrm{C}$, which allowed us to measure subcutaneous temperature $\left(T_{\mathrm{sc}}\right)$ lower than $30^{\circ} \mathrm{C}$. Any $T_{\text {sc }}$ lower than $24^{\circ} \mathrm{C}$ was indicated on the reader as "Low". These transponders allowed for remote monitoring of $T_{\mathrm{sc}}$ and torpor use during acclimation. Daily controls of hamster $T_{\mathrm{sc}}$ began four weeks after implantation. Between 10:30 and 14:30 (2 to $4 \mathrm{~h}$ after lights on), at a random time, we read $T_{\mathrm{sc}}$ with a remote reader (HPR plus, Biomark, Boise, ID, USA) and additionally noted if animal were in characteristic torpid ball-shape posture and whether they responded to gentle opening of the cage. We classified individuals as white, if we observed at least stage 3 of winter fur according to Figala scale (where 1 is dark grey with black stripe on a back and 6 is white without a stripe [91]). We did not score the intensity of fur color change any further. To assess $m_{\mathrm{b}}$ changes under SP, hamsters were weighed (to the nearest $0.1 \mathrm{~g}$; Scout Pro 200, Ohaus, USA) every week during first 16 weeks of acclimation to SP and then every one or two weeks until week 40. In some individuals, socalled "late responders", winter traits may appear even after 32-37 weeks under SP [11]. Hence, all hamsters that did not develop either white fur or torpor use after 16 weeks were maintained under SP up to $\sim 40$ weeks.

\section{Measurement of basal metabolic rate}

Basal metabolic rate was measured in normothermic hamsters at the age of three months. In groups I and IV (Fig. 1), which were acclimated to LP for 5 months, BMR was additionally measured at the age of five months, just before transfer to SP. Then, BMR was measured in all animals again after 16 weeks under SP. In late responders BMR was measured only if we observed torpor use for the first time after 20 weeks in SP and this measurement was done as soon as torpor was observed. Measurements were always done in a repetition, 7 days apart, to assess repeatability of BMR. Animals were weighed before and after each metabolic measurement.

Basal metabolic rate was measured by indirect calorimetry using an open-flow respirometry system (Sable Systems International, Las Vegas NV, USA; henceforth: SSI), as described in Jefimow et al. [92]. Measurements were done in the thermoneutral zone of Siberian hamster $\left(T_{\mathrm{a}} \sim\right.$ $28.5^{\circ} \mathrm{C}$; [93]) and gas exchange was measured for $\sim 7 \mathrm{~h}$ which is long enough to ensure post-absorptive state in this species [93]. BMR was calculated as a rate of the most stable $2 \mathrm{~min}$ of $\mathrm{O}_{2}$ consumption during last three hours of the test. Animals were sealed in $0.85 \mathrm{~L}$ chambers made of polypropylene food containers (HPL 808, Lock\&Lock, Hana Cobi, South Korea) which were placed in a temperature-controlled cabinet (ST-1200 BASIC, PolEko-Aparatura, Wodzisław Śląski, Poland). We measured respiratory gas exchange of 14 animals simultaneously, using two parallel respirometry systems. Air was pulled from outside the building using an air pump (DOA-P501BN, Gast Manufacturing INC., Michigan, USA) and dried in columns of silica gel. The main air stream was split into chambers and a reference gas stream. We regulated flow rate at $\sim 500 \mathrm{ml} \mathrm{min}^{-1}$ upstream of each respirometry chamber using a precise needle valve. After air left chambers, a computer-controlled multiplexer (Intelligent Multiplexer V3, SSI) sequentially selected gases from each chamber. Gas exchange of each animal was measured for 5 min every $44 \mathrm{~min}$ with reference gas concentration readings every $20 \mathrm{~min}$. Air flow was measured downstream of animal chambers using mass flow meter (FlowBar-8, SSI). Then the air stream was subsampled at $\sim 200 \mathrm{ml} \mathrm{min}^{-1}$ and water vapor pressure of the subsampled air was measured with a water vapor analyzer (RH-300, SSI). Air was then dried using a nafion dryer tube (product number 17049, VacuMed, Ventura, CA, USA) embedded in silica gel, and a column of magnesium perchlorate (product number 11636.36, VWR International, Gdańsk, Poland). Subsequently, concentrations of $\mathrm{CO}_{2}$ and $\mathrm{O}_{2}$ were measured. In one system, we measured both rates of $\mathrm{O}_{2}$ consumption $\left(\dot{V} O_{2}\right)$ and $\mathrm{CO}_{2}$ production $\left(\dot{V} C_{2}\right)$. In the second system, only $\dot{V} \mathrm{O}_{2}$ was measured. We used $\mathrm{O}_{2}$ (FC10a, SSI) and $\mathrm{CO}_{2}$ (CA-10, SSI) analyzers. All elements of the respirometry system were connected to PC via an analog-to-digital interface (UI2, SSI). Data were acquired using ExpeData software (SSI) at $0.5 \mathrm{~Hz}$.

Metabolic rate $(\mathrm{MR}, W)$ was calculated using oxyjoule equivalent calculated after Lighton et al. [94]:

$$
\operatorname{MR}(W)=\frac{\dot{V} O_{2}(16+5.164 \times R E R)}{60},
$$

where $\mathrm{VO}_{2}$ was the oxygen consumption $\left(\mathrm{ml} \mathrm{O}_{2} \min ^{-1}\right)$ and $R E R=\frac{\dot{V} C_{2}}{\dot{V} O_{2}}$. Because we did not measure $\dot{V C O}_{2}$ in 
one of the systems, we used RER $=0.80$ which leads to smallest error in the calculation of energy expenditure [95].

\section{Statistical analysis}

Statistical modeling was done in $\mathrm{R}$ using packages lme4 [96] and stats [97], and package emmeans [98] for post-hoc comparisons of estimated marginal means. Initial maximal models were built using ecologically relevant explanatory variables and their interactions, which follows the Regression with Empirical Variable Selection approach [99]. We also used second-order Akaike information criteria (AICc) for post-hoc model comparisons (MuMIn [100]). All results are presented as estimated marginal means $\pm \mathrm{SE}$ compared pairwise with Tukey's HSD test adjusted for multiple comparisons [98]. Statistical significance was accepted at $P \leq 0.050$.

First, we compared life history traits of individuals from all experimental groups. Up to 32 day of life $m_{\mathrm{b}}$ of individuals was determined as the mass of whole litter divided by litter size. Between 45 and 90 days of life all hamsters were weighed approximately once a week ( \pm three days). Analysis of life history traits was done in three steps: 1) analysis of $m_{\mathrm{b}}$ measured before separation of siblings (growth rate between birth and day 32 ); 2) analysis of $m_{\mathrm{b}}$ between days 45 and 90; 3) analysis of $m_{\mathrm{b}}$ just prior to transfer to SP. The first two analyses were done using linear mixed-effect modeling (LME; lme4 [96]) with type III Sums of Squares. The last analysis was done using general linear model (GLM; stats [97]).

In the model which tested the effect of litter order on offspring $m_{\mathrm{b}}$ up to 32 day of life, parental ID was included as a random factor to control for repeated measurements. The final model included litter order (first or third litter), offspring age, litter size, $m_{\mathrm{b}}$ of dam, and litter order $\times$ age and litter order $\times$ litter size interactions (Table 1). In analysis of offspring $m_{\mathrm{b}}$ between $\sim 45$ and $\sim 90$ day of life, animal ID was used as a random factor, and litter order, sex and age were used as fixed factors. The best model also included interactions of age $\times$ litter order and litter order $\times$ sex (Table 1$)$. In the analysis that compared $m_{\mathrm{b}}$ of individuals acclimated to LP for 3 (groups II and III) and 5 months (groups I and IV) prior to transfer to SP, litter order, duration of LD acclimation, and sex were included as fixed factors and the final model included only main effects of these variables (Table 1).

To test the effect of litter order and duration of LP acclimation on offspring photoresponsiveness we used contingency tables with Pearson's $\chi^{2}$ test (stats [97]). To do so we defined categories of torpor use and molting. Molting categories included white individuals if they molted after 16 weeks under SP or grey if they did not molt at all. A separate category included individuals that presented late response and molted later than after 20 weeks under SP. Torpor categories comprised individuals using or not using torpor within 16 weeks of acclimation to SP and individuals using torpor later than after 20 weeks in SP. Effects of litter order and duration of LP acclimation were tested separately. We tested also for the effect of interaction of the above factors on molting and torpor use using Cochran-Mantel-Haenszel test (stats [97]).

To assess the effect of litter order and duration of LP acclimation on $m_{\mathrm{b}}$ changes triggered by SP we used GLM (stats [97]). We calculated proportional change of $m_{\mathrm{b}}$ using last measurement taken in LP as the initial $m_{\mathrm{b}}$. Litter order, duration of LP acclimation, sex, categories of torpor use and molting were included as fixed factors and initial $m_{\mathrm{b}}$ as a covariate. The final model included all factors and interactions of sex $x$ torpor use and sex $x$ molting (Table 1).

Changes of $m_{\mathrm{b}}$ between 16th and 40th week of acclimation to SP in late responding individuals are presented descriptively because these individuals differed in winter traits and also in timing of their development.

To test weather polymorphism of winter phenotype among littermates was affected by litter order we used contingency tables with Pearson's $X^{2}$ test. We classified litters as homogenous if all littermates presented the same phenotype and heterogeneous if littermates presented varied phenotypes.

To test for the effect of litter order and time spent in LP on BMR we used LME (lme4 [96]). We did three separate analyses: 1) to test for the effect of litter order on BMR in 3-months-old animals, 2) to test for the effect of litter order and duration of LP acclimation on changes in BMR triggered by photoperiod, and 3) to analyze changes in BMR in late responding individuals. All models included animal ID and trial as random factors that allowed to control for repeated measurements. Body mass was always included as covariate. The analysis of BMR in 3-months old animals included litter order and sex as fixed factors and their interaction (Table 4). To test for the effect of experimental group and development of winter traits on photoperiod-triggered changes in BMR we used litter order, duration of LP acclimation, photoperiod, sex, and winter traits (molting and torpor use) as fixed factors. The final model included fixed factors except torpor use and all interactions between duration of LP acclimation, photoperiod and molting and between litter order, photoperiod and molting (Table 4). In analysis of further changes of BMR in late responding individuals litter order, duration of LP acclimation, sex, molting and time of measurement (in LP, in SP 
and later in SP) were included as fixed factors. The final model included all main effects and interaction of litter order and time of measurement (Table 4).

\section{Abbreviations}

BMR: Basal metabolic rate; LP: Long photoperiod; $m_{\mathrm{b}}$ : Body mass; SP: Short photoperiod

\section{Acknowledgments}

We thank Annika Herwig and Justin Boyles for critical reading and commenting on the manuscript, and Agnieszka Mazurek, Anna Nowak, Weronika Jadach and Anna Kowalczewska for their help during this study. The study was supported by the grant of National Science Centre, Poland \#2017/25/N/NZ8/00732 awarded to ASPP.

\section{Consent for participate}

Not applicable

\section{Authors' contributions}

ASPP- concept of study, data acquisition, analysis and interpretation of data, writing the first draft of the manuscript and revisions. MJ - data acquisition, revision of the manuscript. MSW - help with data analysis, revision of the manuscript. All authors read and approved the final manuscript.

\section{Funding}

This study was founded by National Science Center, Poland \#2017/25/N/ NZ8/00732.

\section{Availability of data and materials}

The datasets used and/or analysed during the current study are available from the corresponding author on reasonable request. Code will be available upon request to the authors.

\section{Declarations}

\section{Ethics approval and consent to particpate}

All experimental procedures were approved by the Local Committee for Ethics in Animal Research in Bydgoszcz, Poland (decisions: 8/2018, 22/2018, 48/2018, 18/2019)

\section{Consent for publication}

All authors agreed to be listed and approved the manuscript and this submission.

\section{Competing interests}

The authors declare that they have no conflict of interest.

\section{Author details}

'Department of Vertebrate Zoology and Ecology, Nicolaus Copernicus University, Toruń, Poland. ${ }^{2}$ Department of Animal Physiology and Neurobiology, Nicolaus Copernicus University, Toruń, Poland.

\section{Received: 23 October 2020 Accepted: 21 February 2021}

Published online: 18 March 2021

\section{References}

1. Broussard DR, Robertson JL, Evans TJ, Faucher GK, Semanik MG, Heideman PD. Age-related decline in reproductive sensitivity to inhibition by short photoperiod in Peromyscus Leucopus. J Mammal. 2009;90(1):32-9.

2. Kaseloo PA, Crowell MG, Jones JJ, Heideman PD. Variation in basal metabolic rate and activity in relation to reproductive condition and photoperiod in white-footed mice (Peromyscus leucopus). Can J Zool. 2012; 90(5):602-15.

3. Kaseloo PA, Crowell MG, Heideman PD. Heritable variation in reaction norms of metabolism and activity across temperatures in a wild-derived population of white-footed mice (Peromyscus leucopus). J Comp Physiol B. 2014:184(4):525-34.

4. Hoffmann K. The influence of photoperiod and melatonin on testis size, body weight and pelage colour in the Djungarian hamster. J Comp Physiol. 1973;85:267-82.
5. Moffatt CA, Devries AC, Nelson RJ. Winter adaptations of male deer mice (Peromyscus-Maniculatus) and prairie voles (Microtus-Ochrogaster) that vary in reproductive responsiveness to photoperiod. J Biol Rhythm. 1993;8(3):221-32.

6. Bernard DJ, Losee-Olson S, Turek FW. Age-related changes in the photoperiodic response of Siberian hamsters. Biol Reprod. 1997;57(1):172-7.

7. Freeman DA, Goldman BD. Evidence that the circadian system mediates photoperiodic nonresponsiveness in Siberian hamsters: the effect of running wheel access on photoperiodic responsiveness. J Biol Rhythm. 1997;12(2):100-9.

8. Freeman DA, Goldman BD. Photoperiod nonresponsive Siberian hamsters: effect of age on the probability of nonresponsiveness. J Biol Rhythm. 1997; 12(2):110-21.

9. Hart JS. Seasonal changes in insulation of the fur. Can J Zool. 1965;34:53-7.

10. Kuhlmann MT, Clemen G, Schlatt S. Molting in the Djungarian hamster (Phodopus sungorus Pallas): seasonal or continuous process? J Exp Zool A Comp Exp Biol. 2003;295(2):160-71.

11. Lynch GR, Puchalski W. Effect of prolonged short day exposure on thermoregulation in the djungarian hamster (Phodopus sungorus). Living in the Cold: Physiological and Biochemical Adaptations. New York: Elsevier Sciensce Publishing; 1986.

12. Geiser F, Ruf T. Hibernation versus daily torpor in mammals and birds physiological variables and classification of torpor patterns. Physiol Zool. 1995;68(6):935-66.

13. Przybylska AS, Wojciechowski MS, Jefimow M. Physiological differences between winter phenotypes of Siberian hamsters do not correlate with their behaviour. Anim Behav. 2019:147:105-14.

14. Goldman SL, Dhandapani K, Goldman BD. Genetic and environmental influences on short-day responsiveness in Siberian hamsters (Phodopus sungorus). J Biol Rhythm. 2000;15(5):417-28.

15. Goldman SL, Goldman BD. Early photoperiod history and short-day responsiveness in Siberian hamsters. J Exp Zool A Comp Exp Biol. 2003; 296(1):38-45.

16. Gorman MR, Freeman DA, Zucker I. Photoperiodism in hamsters: abrupt versus gradual changes in day length differentially entrain morning and evening circadian oscillators. J Biol Rhythm. 1997;12(2):122-35.

17. Gorman MR, Zucker I. Environmental induction of photononresponsiveness in the Siberian hamster, Phodopus sungorus. Am J Physiol. 1997;272(3 Pt 2): R887-95.

18. Zimova M, Mills $L S$, Nowak JJ. High fitness costs of climate change-induced camouflage mismatch. Ecol Lett. 2016;19(3):299-307.

19. Mills LS, Bragina EV, Kumar AV, Zimova M, Lafferty DJR, Feltner J, et al. Winter color polymorphisms identify global hot spots for evolutionary rescue from climate change. Science. 2018;359(6379):1033-6.

20. Atmeh K, Andruszkiewicz A, Zub K. Climate change is affecting mortality of weasels due to camouflage mismatch. Sci Rep. 2018;8(1):7648.

21. Hut RA, Dardente H, Riede SJ. Seasonal timing: how does a hibernator know when to stop hibernating? Curr Biol. 2014:24(13):R602-5.

22. Dardente H, Wood S, Ebling F, Saenz de Miera C. An integrative view of mammalian seasonal neuroendocrinology. J Neuroendocrinol. 2019;31(5): e12729.

23. Dardente $H$, Hazlerigg DG, Ebling FJ. Thyroid hormone and seasonal rhythmicity. Front Endocrinol (Lausanne). 2014;5:19.

24. Korf HW. Signaling pathways to and from the hypophysial pars tuberalis, an important center for the control of seasonal rhythms. Gen Comp Endocrinol. 2018;258:236-43.

25. Johnston JD, Ebling FJ, Hazlerigg DG. Photoperiod regulates multiple gene expression in the suprachiasmatic nuclei and pars tuberalis of the Siberian hamster (Phodopus sungorus). Eur J Neurosci. 2005;21(11):2967-74.

26. Murphy M, Jethwa PH, Warner A, Barrett P, Nilaweera KN, Brameld JM, et al. Effects of manipulating hypothalamic triiodothyronine concentrations on seasonal body weight and torpor cycles in Siberian hamsters. Endocrinology. 2012;153(1):101-12

27. Ebling FJ. On the value of seasonal mammals for identifying mechanisms underlying the control of food intake and body weight. Horm Behav. 2014; 66(1):56-65

28. Huck UW, Labov JB, Lisk RD. Food restricting young hamsters (Mesocricetus auratus) affects sex ration and growth of subsequent offspring. Biol Reprod. 1986:35:592-8

29. Mitchell GW, Guglielmo CG, Wheelwright NT, Freeman-Gallant CR, Norris DR Early life events carry over to influence pre-migratory condition in a freeliving songbird. PLoS One. 2011;6(12):e28838. 
30. Helle H, Koskela E, Mappes T. Life in varying environments: experimental evidence for delayed effects of juvenile environment on adult life history. J Anim Ecol. 2012;81(3):573-82.

31. Searcy CA, Gray LN, Trenham PC, Shaffer HB. Delayed life history effects, multilevel selection, and evolutionary trade-offs in the California tiger salamander. Ecology. 2014;95(1):68-77.

32. Rogovin KA, Bushuev AV, Khruscheva AM, Vasilieva NY. Resting metabolic rate, stress, testosterone, and induced immune response in spring- and fallborn males of Campbell's dwarf hamsters: maintenance in long-day conditions. Biol Bull Rev. 2014;4(3):181-91.

33. Butler MP, Trumbull JJ, Turner KW, Zucker I. Timing of puberty and synchronization of seasonal rhythms by simulated natural photoperiods in female Siberian hamsters. Am J Physiol Regul Integr Comp Physiol. 2007; 293(1):R413-20.

34. Ergon T, Lambin $X$, Stenseth NC. Life-history traits of voles in a fluctuating population repsond to immediate environment. Nature. 2001;411:1043-5.

35. Butler MP, Zucker I. Seasonal pelage changes are synchronized by simulated natural photoperiods in Siberian hamsters (Phodopus sungorus). J Exp Zool A Ecol Genet Physiol. 2009;311(7):475-82.

36. Clutton-Brock TH, Major M, Albon SD, Guinness FE. Early development and population dynamics in Red Deer. I. Density-dependent effects on juvenile survival. J Anim Ecol. 1987;56(1):53-67.

37. Rose KE, Clutton-Brock TH, Guinness FE. Cohort variation in male survival and lifetime breeding success in red deer. J Anim Ecol. 1998;67(6):979-86.

38. Plard F, Gaillard JM, Coulson T, Hewison AJM, Douhard M, Klein F, et al. The influence of birth date via body mass on individual fitness in a long-lived mammal. Ecology. 2015:96(6):1516-28.

39. Gorman MR, Zucker I. Seasonal adaptations of Siberian hamsters. II. Pattern of change in day length controls annual testicular and body weight rhythms. Biol Reprod. 1995;53:116-25.

40. Butler MP, Turner KW, Park JH, Butler JP, Trumbull JJ, Dunn SP, et al. Simulated natural day lengths synchronize seasonal rhythms of asynchronously born male Siberian hamsters. Am J Physiol Regul Integr Comp Physiol. 2007;293(1):R402-12.

41. Ross PD. Phodopus sungorus. Mamm Species. 1998;595:1-9.

42. Ingram DL, Mandl AM, Zuckerman S. The influence of age on litter-size. J Endocrinol. 1958;17(3):280-5.

43. O'Riain MJ, Jarvis JUM. The dynamics of growth in naked mole-rats: the effects of litter order and changes in social structure. J Zool. 1998;246:49-60.

44. Przybylska AS, Wojciechowski MS, Jefimow M. Photoresponsiveness affects life history traits but not oxidative status in a seasonal rodent. Front Zool. 2019;16(1):11.

45. Koketsu Y, Tani S, lida R. Factors for improving reproductive performance of sows and herd productivity in commercial breeding herds. Porcine Health Manag. 2017;3:1.

46. Rieger JF. Body size, litter size, timing of reproduction, and juvenile survival in the Uinta ground squirrel, Spermophilus armatus. Oecologia. 1996;107(4): 463-8.

47. Rodel HG, Prager G, Stefanski V, von Holst D, Hudson R. Separating maternal and litter-size effects on early postnatal growth in two species of altricial small mammals. Physiol Behav. 2008;93(4-5):826-34.

48. Milligan BN, Fraser D, Kramer DL. Within-litter birth weight variation in the domestic pig and its relation to pre-weaning survival, weight gain, and variation in weaning weights. Livest Prod Sci. 2002;76(1-2):181-91.

49. Myers P, Master LL. Reproduction by Peromyscus-Maniculatus - size and compromise. J Mammal. 1983;64(1):1-18.

50. Prendergast BJ, Kriegsfeld LJ, Nelson RJ. Photoperiodic polyphenisms in rodents: neuroendocrine mechanisms, costs, and functions. Q Rev Biol. 2001;76(3):293-325.

51. Boratyński JS, Jefimow M, Wojciechowski MS. Phenotypic flexibility of energetics in acclimated Siberian hamsters has a narrower scope in winter than in summer. J Comp Physiol B. 2016;186(3):387-402.

52. Mahlert B, Gerritsmann H, Stalder G, Ruf T, Zahariev A, Blanc S, et al. Implications of being born late in the active season for growth, fattening, torpor use, winter survival and fecundity. Elife. 2018;7:e31255.

53. Sadowska J, Gębczyński AK, Paszko K, Konarzewski M. Milk output and composition in mice divergently selected for basal metabolic rate. J Exp Biol. 2015;218(Pt 2):249-54.

54. Fischbeck KL, Rasmussen KM. Effect of repeated reproductive cycles on maternal nutritional status, lactational performance and litter growth in ad libitum-fed and chronically food-restricted rats. J Nutr. 1987;117(11):1967-75.
55. Havelka MA, Millar JS. Maternal age drives seasonal variation in litter size of Peromyscus leucopus. J Mammal. 2004;85(5):940-7.

56. Fuchs S. Optimality of parental investment - the influence of nursing on reproductive success of mother and female young house mice. Behav Ecol Sociobiol. 1982;10(1):39-51.

57. Edwards HE, Tweedie CJ, Terranova PF, Lisk RD, Wynne-Edwards KE. Reproductive aging in the Djungarian hamster, Phodopus campbelli. Biol Reprod. 1998;58(3):842-8.

58. Lim JN, Senior AM, Nakagawa S. Heterogeneity in individual quality and reproductive trade-offs within species. Evolution. 2014;68(8):2306-18.

59. Cameron H, Monro K, Malerba M, Munch S, Marshall D. Why do larger mothers produce larger offspring? A test of classic theory. Ecology. 2016;97(12):3454-9.

60. Monaghan P. Early growth conditions, phenotypic development and environmental change. Philos Trans R Soc Lond Ser B Biol Sci. 2008; 363(1497):1635-45.

61. Prevot-Julliard AC, Henttonens H, Yoccoz NG, Stenseth NC. Delayed maturation in female bank voles: optimal decision or social constraint? J Anim Ecol. 1999;68:684-97

62. Stumpfel S, Bieber C, Blanc S, Ruf T, Giroud S. Differences in growth rates and pre-hibernation body mass gain between early and late-born juvenile garden dormice. J Comp Physiol B. 2017;187(1):253-63.

63. Michel ES, Demarais S, Strickland BK, Wang G. Birth date promotes a tortoise or hare tactic for body mass development of a long-lived male ungulate. Oecologia. 2018;186(1):117-28.

64. Metcalfe NB, Monaghan P. Compensation for a bad start: grow now, pay later? Trends Ecol Evol. 2001;16(5):254-60.

65. Bertram DF, Chambers RC, Leggett WC. Negative correlations between larval and juvenile growth-rates in winter flounder - implications of compensatory growth for variation in size-at-age. Mar Ecol Prog Ser. 1993; 96(3):209-15.

66. Auer SK, Arendt JD, Chandramouli R, Reznick DN. Juvenile compensatory growth has negative consequences for reproduction in Trinidadian guppies (Poecilia reticulata). Ecol Lett. 2010;13(8):998-1007.

67. Newkirk KD, McMillan HJ, Wynne-Edwards KE. Length of delay to birth of a second litter in dwarf hamsters (Phodopus): evidence for post-implantation embryonic diapause. J Exp Zool. 1997;278(2):106-14.

68. Blumstein DT, Arnold W. Ecology and Social Behavior of Golden Marmots (Marmota caudata aurea). J Mammal. 1998;79(3):873-86.

69. Heldmaier G, Steinlechner S. Seasonal control of energy requirements for thermoregulation in the Djungarian hamster (Phodopus sungorus), living in natural photoperiod. J Comp Physiol. 1981;142(4):429-37.

70. Scholander PF, Hock R, Walters V, Irving L. Adaptation to cold in arctic and tropical mammals and birds in relation to body temperature, insulation, and basal metabolic rate. Biol Bull. 1950;99(2):259-71.

71. Ben-Jonathan N, Hugo ER, Brandebourg TD, LaPensee CR. Focus on prolactin as a metabolic hormone. Trends Endocrinol Metab. 2006;17(3):110-6.

72. Yahata T., Kuroshima A. Role of prolactin in brown adipose tissue thermogenic activity. In: Milton A.S. (eds) Temperature Regulation. Advances in Pharmacological Sciences. Basel: Birkhäuser; 1994.

73. Heldmaier G, Steinlechner S. Seasonal pattern and energetics of short daily torpor in the Djungarian hamster, Phodopus sungorus. Oecologia. 1981;48(2): 265-70

74. Kirsch R, Ouarour A, Pevet P. Daily torpor in the Djungarian hamster (Phodopus sungorus): photoperiodic regulation, characteristics and circadian organization. J Comp Physiol A. 1991:168(1):121-8.

75. Ruf $\mathrm{T}$, Heldmaier $\mathrm{G}$. The impact of daily torpor on energy-requirements in the Djungarian hamster, Phodopus-Sungorus. Physiol Zool. 1992;65(5):994-1010.

76. Cubuk C, Bank JH, Herwig A. The chemistry of cold: mechanisms of torpor regulation in the Siberian hamster. Physiology (Bethesda). 2016;31(1):51-9.

77. Ruf T, Stieglitz A, Steinlechner S, Blank JL, Heldmaier G. Cold exposure and food restriction facilitate physiological responses to short photoperiod in Djungarian hamsters (Phodopus sungorus). J Exp Zool. 1993;267(2):104-12.

78. Lynch GR, Wichman HA. Reproduction and thermoregulation in peromyscus: effects of chronic short days. Physiol Behav. 1981;26(2):201-5.

79. Hoffmann $\mathrm{K}$, Illnerova H. Photoperiodic effects in the Djungarian hamster. Rate of testicular regression and extension of pineal melatonin pattern depend on the way of change from long to short photoperiods. Neuroendocrinology. 1986;43(3):317-21.

80. Heideman PD, Bronson FH. Characteristics of a genetic polymorphism for reproductive photoresponsiveness in the white-footed mouse (Peromyscus leucopus). Biol Reprod. 1991;44(6):1189-96. 
81. Kliman RM, Lynch GR. Evidence for independence of circadian characters and extent of photoresponsiveness in the Djungarian hamster, Phodopus sungorus. J Biol Rhythms. 1991;6(2):159-66.

82. Nelson RJ. Photoperiod-nonresponsive morphs: a possible variable in microtine population-density fluctuations. Am Nat. 1987;130(3):350-69.

83. Ford EB. Polymorphism. Biol Rev Camb Philos Soc. 1945;20(2):73-88.

84. Wynne-Edwards KE. Evolution of parental care in Phodopus: conflict between adaptations for survival and adaptations for rapid reproduction. Am Zool. 1998;38(1):238-50.

85. Lynch EWJ, Coyle CS, Stevenson TJ. Photoperiodic and ovarian steroid regulation of histone deacetylase 1, 2, and 3 in Siberian hamster (Phodopus sungorus) reproductive tissues. Gen Comp Endocrinol. 2017;246:194-9.

86. Banks R, Delibegovic M, Stevenson TJ. Photoperiod- and Triiodothyroninedependent regulation of reproductive neuropeptides, Proinflammatory cytokines, and peripheral physiology in Siberian hamsters (Phodopus sungorus). J Biol Rhythm. 2016;31(3):299-307.

87. Boratyński JS, Jefimow M, Wojciechowski MS. Individual differences in the phenotypic flexibility of basal metabolic rate in Siberian hamsters are consistent on short- and long-term timescales. Physiol Biochem Zool. 2017; 90(2):139-52.

88. Wade GN, Bartness TJ. Effects of photoperiod and gonadectomy on food intake, body weight, and body composition in Siberian hamsters. Am J Phys. 1984;246(1 Pt 2):R26-30.

89. Bartness TJ, Clein MR. Effects of food deprivation and restriction, and metabolic blockers on food hoarding in Siberian hamsters. Am J Physiol Regul Integr Comp Physiol. 1994:266:1111-7.

90. Boratyński JS, Jefimow M, Wojciechowski MS. Melatonin attenuates phenotypic flexibility of energy metabolism in a photoresponsive mammal, the Siberian hamster. J Exp Biol. 2017;220(Pt 17):3154-61.

91. Figala J, Hoffmann K, Goldau G. Zur Jahresperiodik beim Dsungarischen Zwerghamster Phodopus sungorus Pallas. Oecologia. 1973;12:89-118.

92. Jefimow M, Przybylska-Piech AS, Wojciechowski MS. Predictive and reactive changes in antioxidant defence system in a heterothermic rodent. J Comp Physiol B. 2020;190(4):479-92.

93. Gutowski JP, Wojciechowski MS, Jefimow M. Diet affects resting, but not basal metabolic rate of normothermic Siberian hamsters acclimated to winter. Comp Biochem Physiol A Mol Integr Physiol. 2011;160(4):516-23.

94. Lighton JRB, Bartholomew GA, Feener DH. Energetics of locomotion and load carriage and a model of the energy-cost of foraging in the leaf-cutting ant Atta-Colombica Guer. Physiol Zool. 1987;60(5):524-37.

95. Koteja P. Measuring energy metabolism with open-flow Respirometric systems: which design to choose? Funct Ecol. 1996;10:675-7.

96. Bates D, Mächler M, Bolker B, Walker S. Fitting linear mixed-effects models using Ime4. J Stat Softw. 2015;67(1).

97. R Core Team. R: A language and environment for statistical computing. Vienna: R Foundation for Statistical Computing; 2020. https://www.R-project. org/. Accessed Sept 2020.

98. Lenth R. emmeans: Estimated Marginal Means, aka Least-Squares Means. R package version 1.4.6. ed: CRAN; 2020. p. https://CRAN.R-project.org/packa ge=emmeans. Accessed September 2020

99. Goodenough AE, Hart AG, Stafford R. Regression with empirical variable selection: description of a new method and application to ecological datasets. PLoS One. 2012;7(3):e34338.

100. Bartoń K. MuMIn: Multi-Model Inference R package version 1.43.17; 2020,

\section{Publisher's Note}

Springer Nature remains neutral with regard to jurisdictional claims in published maps and institutional affiliations.

Ready to submit your research? Choose BMC and benefit from:

- fast, convenient online submission

- thorough peer review by experienced researchers in your field

- rapid publication on acceptance

- support for research data, including large and complex data types

- gold Open Access which fosters wider collaboration and increased citations

- maximum visibility for your research: over $100 \mathrm{M}$ website views per year

At BMC, research is always in progress.

Learn more biomedcentral.com/submissions 